\title{
E-Health Cross Layer Design
}

\author{
Y.Z. Luan, G.L. Ren \\ Department of Communication Engineering \\ Xidian University \\ China
}

\begin{abstract}
This paper presents a new E-health cross layer design scheme using GA algorithm, each layer protocol is adjusted according to the medical event QoS information flexibly, and ensure the shortest time delay. Using the medical events QoS requirements and existed wireless network facilities to design a new E-health system.
\end{abstract}

Keywords-e-health; cross layer; QoS; GA

\section{INTRODUCTION}

E-health is not a new word. In 1995, some scientists have mentioned that idea. We can also call it m-health. Maybe it can be called artificial intelligent healthcare[1 11]. It requires a number of areas of collaboration. The concept of m-health was first introduced and defined as "mobile computing, medical sensor, and communications technologies for healthcare"[3].

E-health care solutions have promised to transform the whole healthcare process to become more efficient, less expensive and higher quality. In the context of e-Health, numerous flows have generated slightly less than $1 \mathrm{M}$ bytes of data now, and may reach about $12000 \mathrm{M}$ bytes by 2020 . These flows come from various databases such as electronic medical databases, mobilized health databases, personal health databases, mobile health care monitors, genetic sequencing and predictive analytics as well as a large array of biomedical sensors and smart devices. So the design of an efficient Ehealth system is a challenging problem especially for widebandwidth demanding tele-medical applications.

At present, the development of E-health network has many problems to be solved, including the allocation of bandwidth, real-time data transmission, especially in the emergency rescue, low power consumption.

E-health must be work on communication network, and it is well known that communication network has 7seven layers protocol, The OSI (Open System Interconnect Reference Model) defined 7 layers from top to bottom respectively. 7 is application layer, 6 is presentation layer, 5 is session layer, 4 is transport layer, 3 network layer, 2 data link layer( including MAC, medium access control), 1 the physical layer. 4 and upper layers define the functions of the application, including security, management, TDD or not, android or not, the following three layers take charge of flow control, error control, data rate control, routing protocol, access control.

This paper focuses wireless communication cross layer design of E-health network, according to different service QoS level, system choose different transmission scheme, to make the optimal system performance, transport information in time, reduce the power consumption of the device and protect

\author{
P.R. Zhang \\ School of Physics and optoelectronic engineering \\ Xidian University \\ China
}

patients privacy information, etc. The existing wireless network infrastructure such as Wifi, WiMAX or Ad-hoc network can be used as a platform for transmission.

To some extent, existing wireless network standard restrict the development of the E-health system due to the narrow band, coexisted PHY or incompatible MAC definition. To enable true plug-and-play interoperability, all layers of the protocol stack, application profiles, and data exchange formats have to be standardized, which is currently underway in the following groups. The ISO/IEEE 11073 Personal Health Data Working Group defines standards and protocols that facilitate exchange of health information between peripheral area network devices and application hosting devices such as cell phones, personal computers, and PDAs. The group defines transport-independent applications and information profiles including data formats, exchanges, and terminology. Also in E-health system, especially emergency situation, a whole empty wide spectrum specialized for medical communication is needed, because it involves a question of life and death.

In this paper, a new network topology is designed and a new cross-layer design based on the QoS and Genetic Algorithm is described.

\section{NETWORK DESIGN}

In E-health network, doctors and patients communicate by means of electronic equipment through wireless and wired network, do not need face to face. We suppose a PDA(Personal Digital Assistant) is always carried near the patient, and the PDA receives sensor information from the patient, and gives advice to the patient. A patient body may have several sensor modules, the patient has the right to use or not use it. When they do not use the sensor nodes, they can set the sensor nodes in a sleep mode. The system that integrates PDA technology and wireless technology can provide continuous and real-time monitoring of vital signs including heart rate, threedimensional ECG, and SpO. etc. The emergence of PDA as powerful processing and communication platform changes the scope and utility of mobile computers.

As mentioned before, patients communicate with the center network through PDA, each patients' PDA can constitutes a star network with a core or a mesh network without a core. Every core network may constitute upper layer core networks, just like a tree. Fig 1 is a diagram of a new E-health network design. 


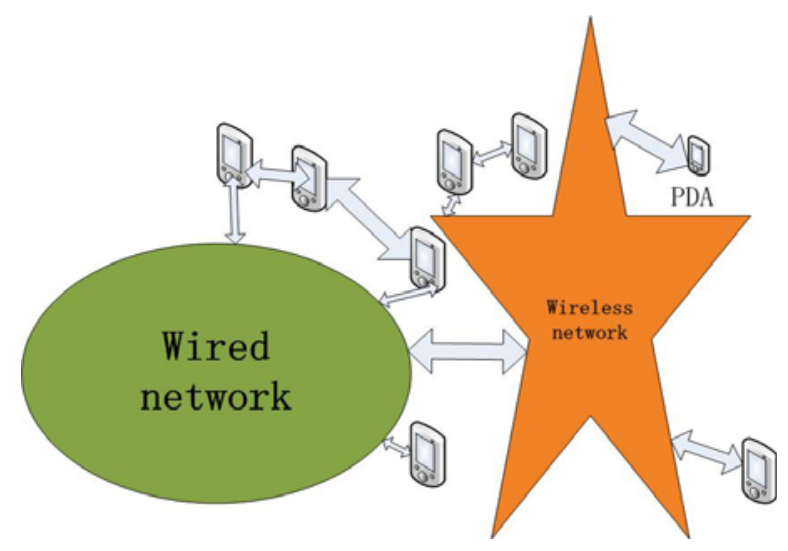

FIGURE I. NEW E-HEALTH NETWORK DESIGN BASED ON EXISTING NETWORKS

We can understand the concept of tree layer network structure as following, the bottom is each sensor node, which is not shown in Fig 1, sensor nodes and PDAs interconnect with one patient to one PDA transmission, and PDAs access wireless network through WiMAX, Bluetooth, or other ad-hoc networks. Then access wired network to the doctor core. The downlink is just the reverse direction.

The E-health applications include the following: wearable systems and personal health-care monitoring, monitoring of soldiers in the battle field, emergency medical care and mass casualty event, home monitoring, computer-assisted rehabilitation and therapy, social networking of relatives and peers of chronically ill patients. each has a different QoS grade, To meet these QoS requirements, the center node should have a suitable mechanism for resource allocation. Next part is the Qos definition.

\section{QOS DEFINED IN E-HEALTH NETWORK}

First we review the various medical service QoS grade. Most of the applications use data rate, delay time, delay time jitter, PSNR(Peak Signal to Noise ratio) packet loss and frame size as basic QoS variables. Table 1 shows some QoS parameters about E-health service. In medical case, there are three situations, emergency, clinical care and home care, In table 2, we define the QoS grade for different situations, number 7 represents the highest priority, number 0 represents the lowest priority.

TABLE I. E-HEALTH QOS SERVICE REQUIREMENTS

\begin{tabular}{|c|l|l|}
\hline E-health service & \multicolumn{1}{|c|}{ Data rate } & \multicolumn{2}{|c|}{ QoS parameters } \\
\hline $\begin{array}{c}\text { Region of Interest } \\
\text { JPEG } \\
\text { Image }\end{array}$ & $\begin{array}{l}15-19 \\
\text { Mbytes }\end{array}$ & $\begin{array}{l}\text { PSNR, Frame } \\
\text { size, } \\
\text { Packet loss }\end{array}$ \\
\hline Radiology & $\sim 6$ Mbytes & $\begin{array}{l}\text { PSNR, Frame } \\
\text { size, } \\
\text { Packet loss }\end{array}$ \\
\hline $\begin{array}{c}\text { Magnetic resonance } \\
\text { imaging (MRI) }\end{array}$ & $<1$ Mbytes & $\begin{array}{l}\text { PSNR, Frame } \\
\text { size, } \\
\text { Packet loss }\end{array}$ \\
\hline Ultrasound video \\
streaming & $250 \mathrm{~Kb} / \mathrm{s}-$ & $\begin{array}{l}\text { PSNR, Frame } \\
\text { Rate, } \\
\text { Frame size, } \\
\text { Packet loss, Delay }\end{array}$ \\
\hline
\end{tabular}

\begin{tabular}{|c|l|l|}
\hline $\begin{array}{c}\text { VideoConference } \\
\text { (Teleconsultation) }\end{array}$ & $>1$ Mbytes & Delay, PSNR \\
\hline $\begin{array}{c}\text { VoiceConference } \\
\text { (Teleconsultation) }\end{array}$ & $\sim 3 \mathrm{~kb} / \mathrm{s}$ & Delay, Packet loss \\
\hline
\end{tabular}

TABLE II. BROADBAND E-HEALTH QOS CLASSIFICATION

\begin{tabular}{|c|l|l|l|}
\hline $\begin{array}{c}\text { Different Medical } \\
\text { Service }\end{array}$ & $\begin{array}{c}\text { Emergency } \\
\text { case }\end{array}$ & $\begin{array}{c}\text { Clinical } \\
\text { care case }\end{array}$ & $\begin{array}{c}\text { Homecare } \\
\text { Case }\end{array}$ \\
\hline ROI JPEG Image & 5 & 6 & 5 \\
\hline Radiology & 0 & 6 & 0 \\
\hline $\begin{array}{c}\text { Magnetic resource } \\
\text { Imaging(MRI) }\end{array}$ & 0 & 6 & 0 \\
\hline $\begin{array}{c}\text { Ultrasound Video } \\
\text { Streaming }\end{array}$ & 7 & 7 & 5 \\
\hline $\begin{array}{c}\text { VideoConference } \\
\text { (Teleconsultation) }\end{array}$ & 2 & 5 & 3 \\
\hline $\begin{array}{c}\text { VoiceConference } \\
\text { (Teleconsultation) }\end{array}$ & 4 & 5 & 5 \\
\hline
\end{tabular}

In table 1, the PSNR is defined as equation (1), it is mainly defined for the image resolution, a value of $35 \mathrm{~dB}$ was recommended from earlier clinical evaluation study to give better medical diagnosis quality[10].

$$
P S N R=10 \log _{10}\left[\frac{255 * 255}{\frac{1}{N M} \sum_{i=0}^{N-1} \sum_{j=0}^{M-1}(x(i, j)-y(i, j))^{2}}\right]
$$

where $x(i, j)$ refers to the pixel $(i, j)$ in the original image and $y(i, j)$ to the pixel $(i, j)$ in the test image. Both images are of size $N * M$.

\section{CROSS-LAYER DESIGN}

Public E-health is effective only if subjects' vital signs are reported to the medical center promptly and reliably at all times regardless of the diverse environments such as streets, homes, offices, shopping malls, etc., in which subjects may be located. Low latency is a requirement of E-health services, aimed at prediction and prevention of life threatening health conditions before they occur. For example, a patient encountered a car accident, and his PDA help him call an ambulance to hospital, now timely transfer image is indispensable. During the video conference session the special surgeon has the ability to preview the injury from different angles through streaming video, to access high resolution images, and communicate through video, voice and text with the attendant orthopedist.

The cross-layer design, is called CLD simply, refers to protocol and model architecture design, which exploit the dependence between layers to obtain optimum gains[2]. In general, cross-layer systems perform three major tasks: data abstracting, optimizing, and reconfiguration. Abstraction and reconfiguration strictly depend on the system model and the interaction.

To achieve this, we present a new cross-layer approach based on a GA (Genetic algorithm) for real-time medical video streaming on existed wireless networks. The process of 
optimizing in cross layer is based on GA, the objective function can be defined as:

$$
f\left(x_{1}, x_{2}, x_{3}\right)=\max \left[\frac{1}{T\left(x_{1}, x_{2}, x_{3}\right)-\sum_{l=1}^{7} t_{l}\left(x_{1}, x_{2}, x_{3}\right)}\right]
$$

where $x_{1}, x_{2}, x_{3}$ is the adjusting parameters in each layer, such as code rate, power, frame rate, etc. $t_{l}$ is the time delay of $l^{\text {th }}$ layer, $T\left(x_{1}, x_{2}, x_{3}\right)$ is the objective whole delay time. With different QoS grade number, different $T\left(x_{1}, x_{2}, x_{3}\right)$ is set forward.

Fig 3 is each layer parameters to be adjusted, it help us understand the idea more clearly.

It is very sorry that the simulating results are still on going. They will be shown in next paper.

\section{CONCLUSION}

This paper suggests using GA as the cross layer optimizer to adjust the processing time according to different QoS requirements in E-health wireless networks.

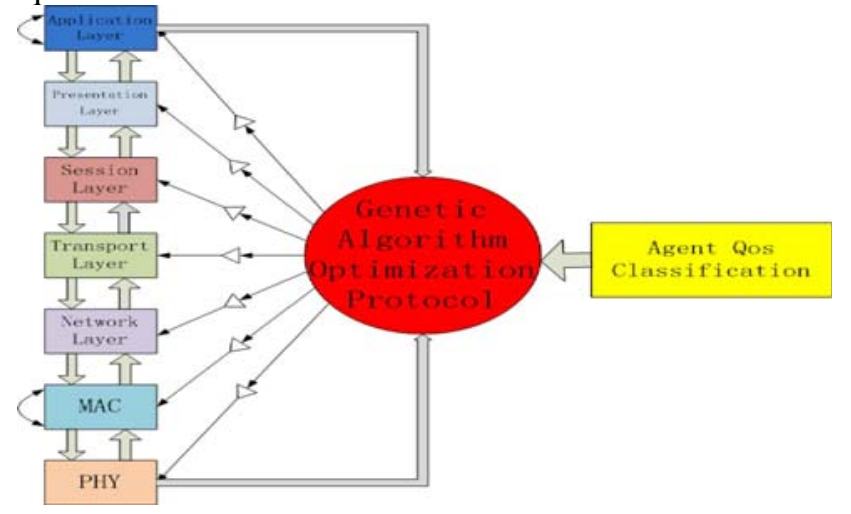

FIGURE II. CROSS LAYER DESIGN DIAGRAM
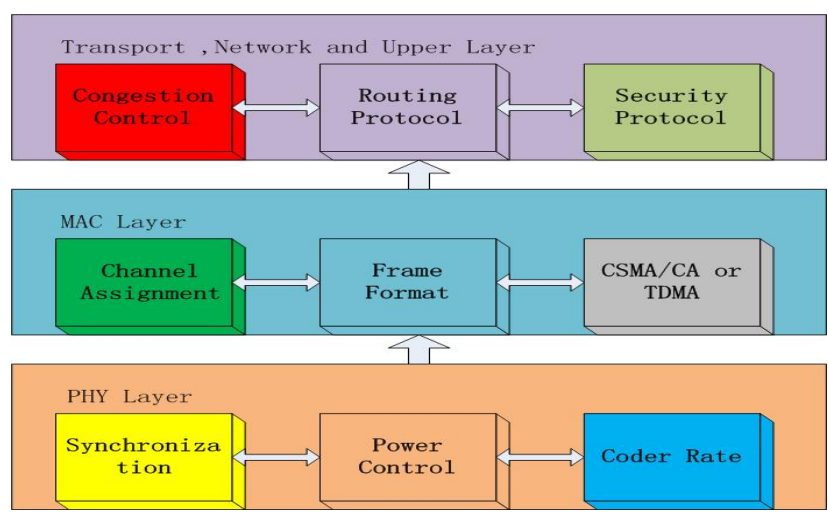

FIGURE III. EACH LAYER DETAIL PARAMETERS

\section{ACKNOWLEDGMENTS}

This work was supported by National Basic Research Program of China (973 Program) under Project 2014CB340205 and National Major Scientific and Technological Project of China (Grant No. 2011ZX03001-007-01).

\section{REFERENCES}

[1] J. Sublett, B. Dempsey and A.C. Weaver, Design and Implementation of a Digital Teleultrasound System for Real-Time Remote Diagnosis, Comp.-Based Med. Sys.,TX, June 1995, pp. 292-99.

[2] Ali Alinejad, Nada Y. Philip and Robert S. H. Istepanian, Cross-Layer Ultrasound Video Streaming Over Mobile WiMAX and HSUPA Networks, IEEE Trans on Inform Tech in Bio, 16(1), pp31-39,Jan 2012

[3] R. S. H. Istepanian, E. Jovanov, and Y. T. Zhang, Guest Editorial Introduction to the Special Section on M-health: Beyond Seamless Mobility for Global Wireless Healthcare Connectivity-Editorial, IEEE Trans. Info.Tech. in Biomed., 8(4), Dec. 2004, pp. 405-14.

[4] S. A. Garawi, R. S. H. Istepanian, and M. A. Abu-Rgheff, 3G wireless communication for mobile robotic tele-ultrasonography systems, IEEE Comms. Mag., vol. 44, no. 4, pp. 91-96, April 2006

[5] Robert S. H. Istepanian, NadaY.Philip and Maria G. Martini, Performance Evaluation of an Enhanced Uplink 3.5G System for Mobile Healthcare Applications, IEEE J.Selected Areas Communication,27(4),pp566-574,2009.

[6] S. Pathak, Basant Kumar, Performance Evaluation of Routing Protocols for sending Healthcare data over WiMAX network. 2014 International Conference on Signal Processing and Integrated Networks (SPIN), pp. 269-274,2014

[7] N. Torabi and V. C. M. Leung, Cross-Layer Design for Prompt and Reliable Transmissions Over Body Area Networks, IEEE J. Biomed. Health Inform. 18(4), pp.1303-1316, Jul. 2014.

[8] M Kathuria, S Gambhir, Quality of Service Provisioning port Layer rotocol for WBAN system, International Conference on Reliability, Optimization and Information Technology(ICROIT), India, ,222-228, Feb 6-8 2014

[9] Marwan Al-Jemeli, and Fawnizu A. Hussin, An Energy Efficient CrossLayer Network Operation Model for IEEE 802.15.4-Based Mobile Wireless Sensor Networks. IEEE Sensors Journal, 15(2), pp684-692, Feb 2014

[10] Maria G. Martini, Robert S.H. Istepanian, Matteo Mazzotti, and Nada Y. Philip, Robust Multilayer Control for Enhanced Wireless Telemedical Video Streaming, IEEE Trans on mobile computing, 9(1) ,pp5-16, JANUARY 2010

[11] Maulin Patel, Jianfeng Wang, Challenges and Prospective in Emerging Body Area Networking Technologies. IEEE Wireless Communications, pp80-88, February 2010 